\title{
Spezielle Trainingstherapie zur Reduktion der Inflammation bei Anti-Jo-1-Syndrom
}

\author{
Special Training Therapy to Reduce Inflammation in Anti-Jo-1 Syndrome
}

Autoren

Institute
T. Greulich', S. Müller ${ }^{3}$, J. Fechtel' , C. Nell' ${ }^{1}$, A. Holland'1', J. P. Bach², B. Tackenberg ${ }^{2}$, H. Schubert ${ }^{4}$, K. Kenn ${ }^{5}$, C. Vogelmeier ${ }^{1}$, A. R. Koczulla ${ }^{1}$

Die Institutsangaben sind am Ende des Beitrags gelistet. $\begin{array}{lll}\text { eingereicht } & \text { 27. 6. } 2011\end{array}$ akzeptiert nach Revision 30. 6. 2011

\section{Bibliografie}

DOI http://dx.doi.org/ 10.1055/s-0030-1256669 Online-Publikation: 24.08.2011

Pneumologie 2011; 65: 624-627

(c) Georg Thieme Verlag KG

Stuttgart · New York

ISSN 0934-8387

Korrespondenzadresse

Dr. A. R. Koczulla

Universitätsklinikum Gießen und Marburg,

Abteilung für Pulmonologie, Standort Marburg Baldingerstraße 1 35042 Marburg koczulla@med.uni-marburg.de

\section{Zusammenfassung \\ $\nabla$}

Wir berichten über den Fall eines 46-jährigen Patienten mit medikamentös behandeltem AntiJo-1-Syndrom. Aufgrund einer progressiven Verschlechterung der Lungenfunktion, Dyspnoe, Abgeschlagenheit und myositischen Beschwerden hatte der Patient bereits systemische Steroide und Azathioprin erhalten. Obwohl hohe Dosen von systemischen Steroiden und Azathioprin gegeben wurden, nahmen die muskuloskelettalen Symptome zu. Der Patient, der sonst regelmäßig Langstreckenläufe von $20 \mathrm{~km}$ Länge unternahm, konnte dieser Form der körperlichen Belastung nicht mehr nachgehen. Wir entschlossen uns, den Patienten mit Hilfe des Galileo ${ }^{\mathrm{TM}}$-Gerätes mit einer regelmäßigen aktiven Muskeltherapie (ausgelöst durch Dehnreflexe) zu behandeln. Vor Beginn und nach drei Monaten regelmäßigen Trainings haben wir folgende Untersuchungen durchgeführt: Messung der Lebensqualität mittels St. Georges Respiratory Questionnaire (SGRQ), ultrasonografische Planimetrie des Musculus quadriceps, 6-Minuten-Gehtest, Lungenfunktionstest sowie die Messung der Serummarker der allgemein-systemischen und muskelspezifischen Inflammation (TNF-alpha, Interleukin-8, CRP, CK, Myoglobin). Unter dem Training mit dem Galileo ${ }^{\mathrm{TM}}-$ Gerät in 5 Trainingseinheiten/Woche kam es innerhalb von zwei Monaten zur deutlichen Besserung des Patienten. Wir führen die Trainingstherapie fort.

\section{Fallbeschreibung}

$\nabla$

Wir behandelten einen 46-jährigen männlichen Patienten mit einem gesicherten Anti-Jo-1-Syndrom mit pulmonalen und neurologischen Beschwerden. Von pulmonaler Seite standen initial Dyspnoe und Husten im Vordergrund. Von neurologischer Seite her dominierten belastungsunabhängige Muskelschmerzen das klinische Bild. Die neurologische Untersuchung war bis auf die Tes-

\section{Abstract \\ $\nabla$}

A 46-year-old patient was frequently seen with a medically treated Anti-Jo- 1 syndrome. The patient had already been treated with azathioprine and oral corticosteroids on account of decreasing lung function, dyspnoea, fatigue, and beginning signs of myositis. Although high doses of steroids and azathioprine were administered, the muscleskeletal syndromes increased steadily. The patient used to be an active long-distance runner $(20 \mathrm{~km})$, but now was unable to perform that kind of physical exercise. It was decided to start a treatment with the Galileo ${ }^{\mathrm{TM}}$ training device for active muscle training of the lower extremities. Before and after three months of training the following assessment was performed: measurement of health-related quality of life (St. Georges respiratory questionnaire, SGRQ), ultrasound measurement of the cross-sectional area of the quadriceps muscle, 6 minute walk test (6 MWT), lung function testing, and assessment of serum markers of inflammation (TNF-alpha, interleukin-8, CRP, CK, myoglobin). After only two months, training with the Galileo ${ }^{\mathrm{TM}}$ five times a week has improved the patient's conditions dramatically. The training will be continued.

tung der peripheren Muskelkraft unauffällig. Hier wurde eine proximal betonte, symmetrische 4/5-Parese der oberen und unteren Extremität festgestellt. Die Muskeleigenreflexe waren nicht eingeschränkt. Vor Behandlungsbeginn lag der Creatininkinase-Wert (CK) bei $6000 \mathrm{U} / \mathrm{l}$, begleitet von einem Anstieg des Myoglobins und der Transaminasen. Darüber hinaus sahen wir eine Leukozytose, eine leichte Erhöhung des CRP sowie einen erhöhten Anti-Jo-Titer. Die distalen Phalan- 

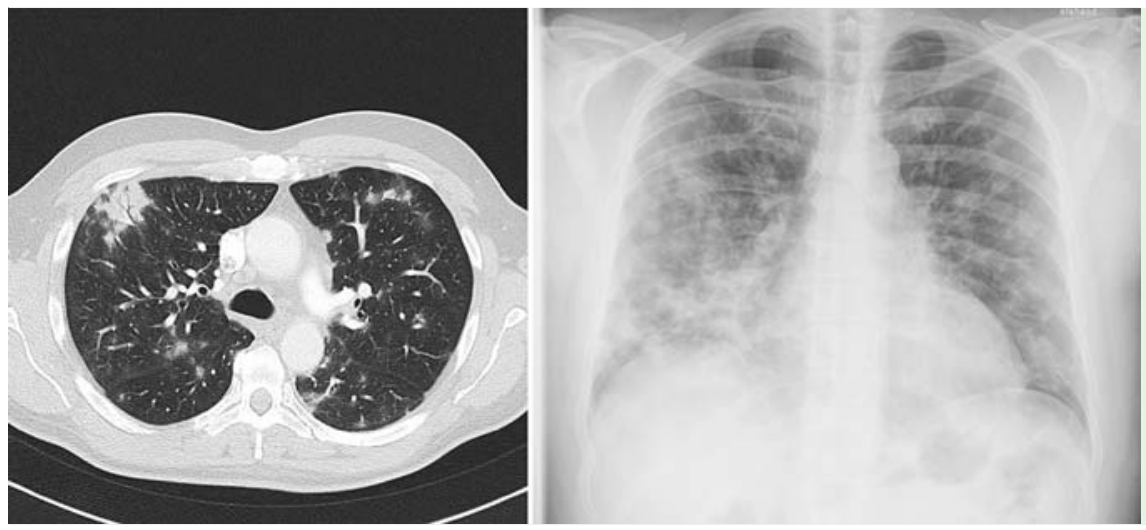

Abb. 1 Röntgen/CT-Bild (eine Schnittebene) des Patienten. Das Röntgenbild zeigt Infiltrate im rechten Unterlappen und einen kleinen Pleuraerguss. In der CT sieht man in der gezeigten Schnittebene multiple, diffus verteilte, alveoläre Infiltrationen mit soliden Knötchen sowie eine Infiltration mit positivem Bronchoaerogramm (links oben).

gen beider Hände offenbarten Zeichen einer leichten Arthritis, andere Hautveränderungen waren nicht erkennbar.

Die Lungenfunktion zeigte eine restriktive Ventilationsstörung mit einer hochgradig eingeschränkten Diffusion ohne Zeichen einer Obstruktion. Die $\mathrm{FEV}_{1}$ lag bei 3,371 (84\%), die IVC betrug 3,961 (76\%), die TLC war 4,67 (63\%), die Hb-korrigierte DLCO war $5,22 \mathrm{mmol} / \mathrm{l} / \mathrm{min} / \mathrm{kPa}(47 \%)$.

Das Röntgenbild zeigte initial Infiltrate im rechten Unterlappen und einen kleinen Pleuraerguss. In der nachfolgend durchgeführten CT sah man multiple, diffus verteilte, alveoläre Infiltrationen mit soliden Knötchen sowie positive Bronchogramme ( $\bullet$ Abb.1). Die mediastinalen Lymphknoten waren deutlich vergrößert. In der Bronchoskopie zeigte sich endoluminal eine unauffällige Schleimhaut sowie keinerlei mukopurulente Sekretion. Die transbronchialen Biopsien ergaben histologisch den Befund einer eher milden chronischen Inflammation ohne den Nachweis von Granulomen. In der bronchoalveolären Lavage fanden wir eine deutliche Lymphozytose (54\%) mit einer deutlich erniedrigten CD4/CD8-Ratio von 0,1. Die mikrobiologische Untersuchung einschließlich der Suche nach Mykobakterien war ergebnislos. Periphere Biopsien wurden nicht durchgeführt.

Es wurde eine Therapie mit systemischen Steroiden begonnen $(1 \mathrm{mg} / \mathrm{kg} \mathrm{Kg} / \mathrm{die})$, von welcher der Patient klinisch zunächst sehr profitierte. Es kam auch zu einem CK-Abfall auf Werte von 250 - 300 U/l, sodass die Dosis schließlich auf $15 \mathrm{mg} /$ die reduziert werden konnte. Im weiteren Verlauf nahmen die Muskelschmerzen (beinbetont) wieder deutlich zu. Konkordant dazu fanden sich auch laborchemisch Zeichen für die wieder aufflammende Myositis sowie eine deutliche Verschlechterung der restriktiven Ventilationsstörung, sodass die medikamentöse Therapie um Azathioprin $150 \mathrm{mg} /$ die erweitert wurde. Hierunter besserte sich zwar die Lungenfunktion des Patienten, die Muskelschmerzen beinbetont persistierten jedoch. Wir entschlossen uns dazu, die Therapie um ein aktives Muskeltraining in der Hocke mittels des Galileo ${ }^{\mathrm{TM}}$-Trainingsgerätes (Novotec Medical, Pforzheim, Deutschland) zu erweitern, und leiteten für den Patienten ein Training auf dem Galileo ${ }^{\mathrm{TM}_{-}}$Gerät ein, das fünfmal wöchentlich (werktags) statt fand ( $\bullet$ Abb.2). Die Ratio hierfür bestand in der Annahme, dass mittels regelmäßiger Bewegungstherapie lokale Inflammation minimiert werden könnte [1]. Als Verlaufsparameter bezüglich der Bewegungstherapie wählten wir den St. Georges Respiratory Questionnaire (SGRQ) als Maß der Lebensqualität, den 6-Minuten-Gehtest, den sonografisch bestimmten Querschnitt des Musculus quadriceps femoris, wie in der Literatur beschrieben [2], sowie Marker der systemischen und muskelspezifischen Inflammation.

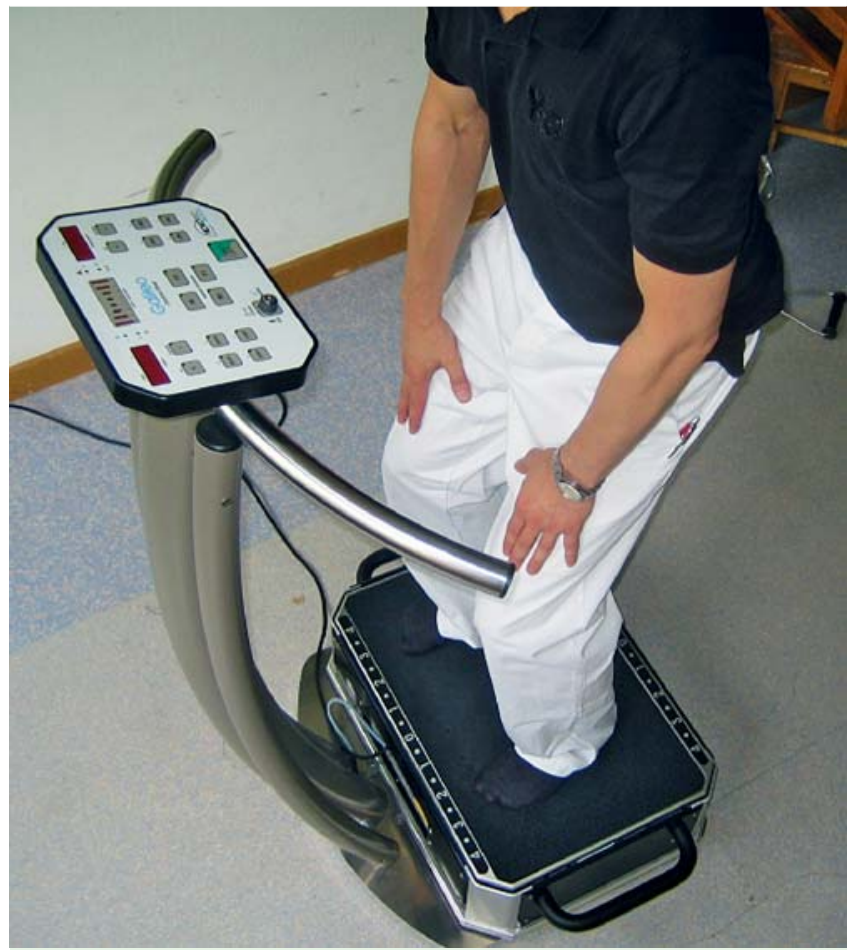

Abb.2 Galileo ${ }^{\mathrm{TM}}$-Gerät mit Abbildung der Fußstellung und die Trainingsstellung in Hocke.

Das Prinzip der vibratorischen Muskelstimulation mit dem Galileo ${ }^{\mathrm{TM}}$-System beruht auf reflektorisch ausgelösten Muskelkontraktionen. Der Trainierende steht dabei auf einer vibrierenden Wippe, die um eine Achse senkrecht zur Körpermitte schwingt. Die Vibrationsplatte löst mit Frequenzen zwischen 5 und $26 \mathrm{~Hz}$ seitenalternierend kleine, nach apikal gerichtete Bewegungen des jeweiligen Beins aus. Dadurch werden reflektorisch neuromuskuläre Reaktionen in den betroffenen Muskeln und am gesamten Haltungsapparat verursacht, die vom Ablauf her den Schritten beim Gehen und Laufen und damit den Grundelementen unserer Fortbewegung entsprechen.

\section{Trainingsplan und weiterer Verlauf unter Therapie $\nabla$}

Der Trainingsaufbau erfolgte nach den bekannten Kriterien der Trainingsgestaltung, also der Drittelung des Trainings in Einleitung (Aufwärmen), Hauptteil (Zielverfolgung) und Schlussteil (Cool down). Die Aufwärmphase bestand aus einem Spaziergang 
von 60-90 Minuten Dauer durch den Wald. Anschließend erfolgte das Galileo ${ }^{\mathrm{TM}}$-Training mit einer Reizfrequenz von zunächst $18 \mathrm{~Hz}$ und einer Fußstellung von 1,5-2,5 Einheiten des Gerätes (je weiter die Fußstellung, desto anstrengender, 1,5 entspricht einer mittleren Fußstellung). Die Gesamtdauer des Reizes betrug zwei Minuten, ebenso die Pausenzeit. Ab der zweiten Einheit wurde mit $18 \mathrm{~Hz}$ und einer gesteigerten Amplitude trainiert, ab der vierten Einheit mit $26 \mathrm{~Hz}$ und Fußstellung 2. Ab Einheit 6 wurde die Fußstellung verbreitert und die Anzahl der Reizsetzung auf fünf erhöht. Im Anschluss an das jeweilige Training erfolgte das Cool down bei $12 \mathrm{~Hz}$ über drei Minuten. Nach 10 Einheiten wurde das Training auf Leistungserhalt umgestellt. Die Einheiten erfolgten drei Wochen lang in der Intensität drei Trainingseinheiten pro Woche mit einer Frequenz von $26 \mathrm{~Hz}$, fünf Durchgänge über zwei Minuten und in Woche 4 fünf Durchgänge über 3 Minuten. Aufwärmen und Cool down waren gleich.

Unter dieser Therapie kam es innerhalb von zwei Monaten bei konstanter medikamentöser Therapie zu einer deutlichen Verbesserung der Symptome und objektiven Untersuchungsbefunde des Patienten. Der Patient gab an, deutlich weniger Schmerzen im Bereich der Extremitätenmuskulatur zu verspüren und sich um ein Vielfaches leistungsfähiger zu fühlen. Die laborchemisch detektierbare Schädigung des Muskels nahm nach einem kurzen initialen Anstieg ab, im Verlauf von 2 Monaten fiel die CK von

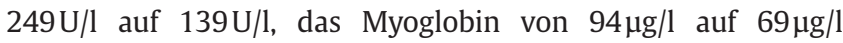
( Abb.3a). Parallel dazu fielen auch die Marker der systemischen Inflammation, IL-8 von $17 \mathrm{ng} / \mathrm{l}$ auf $6.7 \mathrm{ng} / \mathrm{l}$, TNF-alpha von $11 \mathrm{ng} / \mathrm{l}$ auf $8.9 \mathrm{ng} / \mathrm{l}(\bullet \mathrm{Abb} .3 \mathrm{~b}$ ). Die Muskelplanimetrie zeigte einen Muskelzuwachs von $3.6 \mathrm{~cm}^{2}$ auf $10.9 \mathrm{~cm}^{2}(\bullet$ Abb. $3 \mathrm{c}$ ). Im 6-MWT kam es zu einer Verbesserung der Gehstrecke von initial 600 auf $725 \mathrm{~m}$ ( Abb.3c). Die Lebensqualität besserte sich, der SGRQ fiel von 21 auf 10 Punkte, wobei eine Änderung von 4 Punkten bereits als eine klinisch relevante Verbesserung gilt $(\checkmark$ Abb.3d). Die Lungenfunktionsprüfung war bereits vor Beginn des körperlichen Trainings weitgehend normwertig und änderte sich im Verlauf nicht.

\section{Diskussion \\ $\nabla$}

Wir präsentierten den Fall eines Patienten mit Anti-Jo-1-Antikörper-Syndrom, dessen klinischer Verlauf bezüglich der muskulären Probleme durch körperliches Training gebessert werden konnte. Die klinische Besserung ließ sich durch eine Zunahme der Muskelmasse der unteren Extremitäten trotz systemischer Steroidtherapie, durch eine erhöhte Gehstrecke, einen Rückgang der pathologisch erhöhten Muskelenzyme und der systemischen Inflammationsparameter objektivieren.

Physische Aktivität und Inflammation stehen in komplexer Wechselwirkung miteinander [3]. Nach Training mit hohen Intensitäten über einen längeren Zeitraum findet man erhöhte Parameter der systemischen Inflammation [4]. Andererseits reduziert regelmäßige moderate körperliche Aktivität systemische Inflammation [4]. Ein möglicher Mechanismus besteht in der verminderten Expression von Toll-like-Rezeptoren (TLR) auf Monozyten, welche für die Entstehung einer systemischen Inflammation eine entscheidende Rolle spielen [5]. Ein zweiter möglicher Mechanismus besteht in der Hochregulation des Transkriptionsfaktors Peroxisome-proliferator-activated Receptor- $\gamma$ Coactivator $1 \alpha(\mathrm{PGC} 1 \alpha)$ durch regelmäßiges körperliches Training [1]. Mehrere Mechanismen werden diskutiert, mit Hilfe derer PGC1 $\alpha$ zur Inhibierung der systemischen Inflammation beitragen
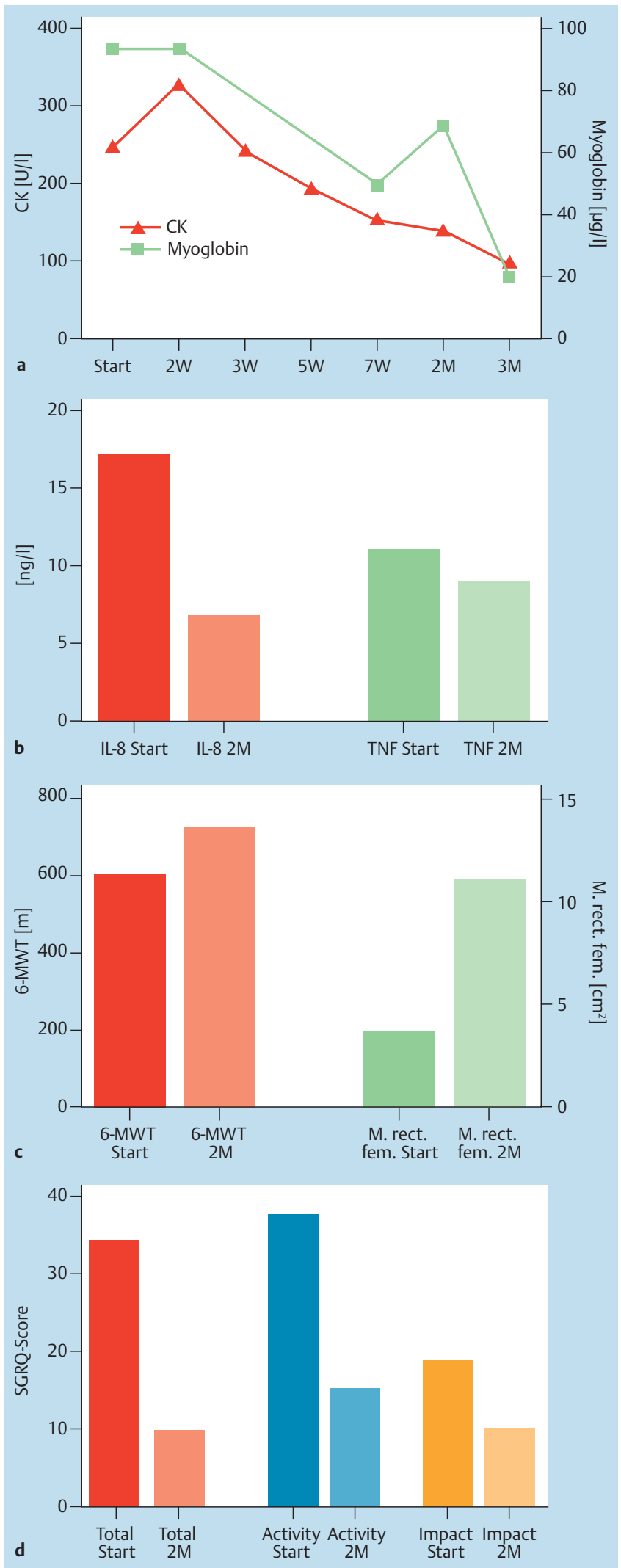

Abb.3 Effekte der Trainingstherapie. Eine zweimonatige aktive Bewegungstherapie hat bei diesem Patienten zu verminderten Markern der muskulären Schädigung (a), zu verminderten systemischen Inflammationsparametern (b), zu einem erhöhten Muskelquerschnitt des M. rect. femoris trotz der systemischen Steroidtherapie (c), zu einer höheren Gehstrecke (c) und zu einer verbesserten Lebensqualität (d) geführt. 
könnte: PGC1 $\alpha$ führt zur Genexpression von Enzymen, welche zur Inaktivierung reaktiver Sauerstoff-Spezies essentiell beitragen [6]. Des Weiteren führt PGC1 $\alpha$ zumindest im Mausmodell zu einer Veränderung des Muskelfaserprofils hin zu Muskelfasern der Typen I und IIa [7]. Hierdurch wird die muskuläre Vaskularisation und Sauerstoffutilisation verbessert. Darüber hinaus bewirkt PGC1 $\alpha$ eine vermehrte Expression mitochondrialer Gene [8]. Zusammenfassend bestehen die Effekte also in einer verbesserten Durchblutung der Muskulatur, einer verbesserten Utilisation von Sauerstoff und einer Inhibierung lokaler Inflammation. Dies würde sich dann im Gesamtorganismus widerspiegeln und zu einer verminderten Ausprägung systemischer Inflammation führen.

Als Ziele der Trainingstherapie haben wir bei unserem Patienten den Muskelaufbau mit dem Synergieeffekt der Durchblutungsförderung und Kraftentwicklung definiert. Im Allgemeinen bedeutet das bezogen auf die Therapie die Wiederherstellung, die Verbesserung und anschließend den Erhalt des erreichten Zustandes. Die Trainingsziele wurden nach den Prinzipien der Trainingslehre für den Patienten formuliert [9]. Im Speziellen wurde das Trainingsprinzip eines langfristigen Leistungsaufbaus angewandt [10]. Hierbei werden trainingswirksame Reize ansteigender Belastung gesetzt (Prinzip der progressiven Belastungssteigerung). Der Reiz muss dabei überschwellig sein. Für ein langfristiges Training ist es notwendig, die Komponenten Intensität, Umfang, Dauer und Häufigkeit unter Berücksichtigung der individuellen Trainingsanpassung zu steigern.

Das Galileo ${ }^{\mathrm{TM}}$-Training erzielt die Trainingseffekte durch Kraft, Koordination und Schnelligkeit. Ein Intervalltraining sorgt für den trainingswirksamen Reiz durch eine lohnende, aber unvollständige Pause, sodass die Reizsetzung in der Summe wirken kann. Nach dem Training erfolgt ein Tag Pause zur Regeneration und Adaptation (Superkompensation). Innerhalb der Einheiten soll es zu einer ansteigenden Belastung kommen, die in Umfang und Intensität gesteigert wird. Die Steigerung erfolgt durch die Frequenz des Reizes (Impulse pro Zeiteinheit) und die Amplitude (Fußstellung). Dabei gilt: Je höher die Frequenz und je größer die Amplitude desto stärker der Kraftaufwand (Novotec, Deutschland). Die simultane Kombination aus Kraft, Koordination und Schnelligkeit könnte gegenüber herkömmlichem Kraft- und Ausdauertraining Vorteile haben, wobei vergleichende Daten fehlen. Sicherlich ist das Galileo ${ }^{\mathrm{TM}}$-Training aufgrund der zeitlichen Vorteile ein interessantes neues Trainingskonzept. Die Verbesserung der Lebensqualität war für den Patienten deutlich, was auch durch den Abfall des SGRQ um 11 Punkte eindrucksvoll wiedergegeben wird. Es scheint logisch, diese Veränderung weitgehend auf den Trainingseffekt (Erhöhung des Muskelquerschnitts, Erhöhung der 6-Minuten-Gehstrecke) zurückzuführen. Die größte Veränderung des SGRQ fanden wir in der Subgruppe Aktivität, wohingegen sich Impact kaum und Symptome (bei völligem Fehlen respiratorischer Symptome vor Beginn der Therapie, entsprechend einem Score von 0) unverändert fanden. Es muss aber betont werden, dass bei einer Einzelfallbetrachtung der Effekt auch rein Placebo-vermittelt sein kann.

Zusammenfassend haben wir einen Patienten mit Anti-Jo-1-Antikörper-Syndrom und massiven muskulären Beschweren mit einer aktiven Bewegungstherapie erfolgreich behandeln können. Die klinische Besserung ließ sich durch eine Zunahme der Muskelmasse der unteren Extremitäten, durch eine erhöhte Gehstrecke, einen Rückgang der pathologisch erhöhten Muskelenzyme und der systemischen Inflammationsparameter objektivieren. Trotz eines inflammatorisch veränderten Muskels kann es also sinnvoll sein, diesen mit moderater Intensität zu trainieren. Hierdurch kann es sogar zu einem Abfall systemischer Inflammationsparameter kommen.

\section{Verwendete Abkürzungen in alphabetischer Reihenfolge:}

6-MWT: 6-Minuten-Gehtest; CK: Creatininkinase; CRP: C-reaktives Protein; DLCO: Diffusionskapazität; $\mathrm{FEV}_{1}$ : Einsekundenkapazität; IL-8: Interleukin-8; IVC: Inspiratorische Vitalkapazität; M. rect. fem.: Musculus rectus femoris; M: Monat; PGC1 $\alpha$ : Peroxisome-proliferator-activated Receptor- $\gamma$ Coactivator $1 \alpha$; SGRQ: St. Georges Respiratory Questionnaire; TLC: Totale Lungenkapazität; TLR: Toll-like Receptor; TNF-Alpha: Tumornekrosefaktoralpha; W: Woche

\section{Interessenkonflikt \\ $\nabla$}

Die Autoren geben an, dass kein Interessenkonflikt besteht.

H. Schubert ist Mitarbeiter von Novotec Medical, Pforzheim, Deutschland.

Institute

${ }^{1}$ Klinik für Innere Medizin, Schwerpunkt Pneumologie, Philipps-Universität Marburg

${ }^{2}$ Klinik für Neurologie, Philipps Universität Marburg

${ }^{3}$ Abteilung für Physiotherapie, Philipps Universität Marburg

${ }^{4}$ Firma Novotec Medical GmbH, Pforzheim

${ }^{5}$ Schön Klinik Berchtesgadener Land, Schönau am Königssee

\section{Literatur}

1 Handschin C, Spiegelman BM. The role of exercise and PGC1alpha in inflammation and chronic disease. Nature 2008; 454: $463-469$

2 Seymour JM, Ward K, Sidhu PS et al. Ultrasound measurement of rectus femoris cross-sectional area and the relationship with quadriceps strength in COPD. Thorax 2009; 64: 418-423

3 Febbraio MA. Exercise and inflammation. J Appl Physiol 2007; 103: $376-377$

4 Gleeson M. Immune function in sport and exercise. J Appl Physiol 2007; 103: $693-699$

5 Gleeson M, McFarlin B, Flynn M. Exercise and Toll-like receptors. Exerc Immunol Rev 2006; 12: 34-53

6 St-Pierre J, Drori S, Uldry M et al. Suppression of reactive oxygen species and neurodegeneration by the PGC-1 transcriptional coactivators. Cell 2006; 127: $397-408$

7 Lin J, Wu H, Tarr PT et al. Transcriptional co-activator PGC-1 alpha drives the formation of slow-twitch muscle fibres. Nature 2002; 418: $797-801$

8 Jager S, Handschin C, St-Pierre J et al. AMP-activated protein kinase (AMPK) action in skeletal muscle via direct phosphorylation of PGC1alpha. Proc Natl Acad Sci U.S.A 2007; 104: 12017-12022

9 Seidenspinner D. Training in der Physiotherapie 4. Aufl. Heidelberg: Springer-Verlag; 2005

10 Weineck J. Optimales Training: Leistungsphysiologische Trainingslehre unter besonderer Berücksichtigung des Kinder- und Jugendtrainings 16. Aufl. Balingen: Spitta; 2009 